\title{
THE CONTINUITY OF THE LYAPUNOV EXPONENT FOR ANALYTIC QUASI-PERIODIC JACOBI OPERATORS
}

\author{
KAI TAO
}

College of Sciences, Hohai University, 1 Xikang Road Nanjing Jiangsu 210098 P.R.China

E-mail address: tao.nju@gmail.com;ktao@hhu.edu.cn

Keywords:Lyapunov Exponent, Jacobi Operators, Weak Liouville

Abstract. In this paper, we use the large deviation theorem and avalanche principle developed by Goldstein and Schlag in [3], to prove that the Lyapunov exponent for analytic quasi-periodic Jacobi operators with weak Liouville frequency is log-Holder continuous.

\section{Introduction}

In this paper we study the continuity of the Lyapunov exponent associated with 1-D quasi-periodic Jacobi operators on $l^{2}(\mathbb{Z})$

$$
\left(H_{x, \omega} \phi\right)(n)=-b(x+(n+1) \omega) \phi(n+1)-b(x+n \omega) \phi(n-1)+a(x+n \omega) \phi(n), \mathrm{n} \in \mathbb{Z},
$$

where $x \in \mathbb{T}, a(x), b(x)$ are analytic on $\mathbb{T}$ and $b(x)$ is not identically zero. $\omega$ is called frequency, which is usually set to be irrational number. Set

$$
\begin{gathered}
\mathrm{A}(\mathrm{x}, \mathrm{E}, \omega)=\frac{1}{b(x+\omega)}\left(\begin{array}{cc}
a(x)-E & -b(x) \\
b(x+\omega) & 0
\end{array}\right) . \\
M_{N}(x, E, \omega)=M_{[1, N]}(x, E, \omega)=A(x+(N-1) \omega, E, \omega) A(x+(N-2) \omega, E, \omega \ldots A(x, E, \omega),
\end{gathered}
$$

Define the analytic matrix

$$
M_{N}^{a}(x, E, \omega)=M_{[1, N]}^{a}(x, E, \omega):=A^{a}(x+(N-1) \omega, E, \omega) A^{a}(x+(N-2) \omega, E, \omega) \ldots A^{a}(x, E, \omega),
$$

where

Set

$$
A^{a}(\mathrm{x}, \mathrm{E}, \omega)=\left(\begin{array}{cc}
a(x)-E & -b(x) \\
b(x+\omega) & 0
\end{array}\right)
$$

$$
L_{N}(E, \omega)=\frac{1}{N} \int_{\mathbb{T}} \log \left\|M_{N}(x, E, \omega)\right\| d x,
$$

and due to the subadditive property, the limits

$$
\mathrm{L}(E, \omega)=\lim _{N \rightarrow \infty} \int_{\mathbb{T}} \frac{1}{N} \log || M_{N}(x, E, \omega)|| d x=\lim _{N \rightarrow \infty} L_{n}(E, \omega) \geq 0,
$$

exists, which is defined to be the Lyapunov exponent. Fixed $\omega \in \mathbb{R} \backslash \mathbb{Q}$, consider the continued fraction expansion $\omega=\left[a_{1}, a_{2}, \cdots\right]$ with convergence $\frac{p_{s}}{q_{s}}$ for $\mathrm{s}=1,2, \ldots$. Let

$$
\beta=\beta(\omega)=\lim _{s} \sup \frac{\log q_{s+1}}{q_{s}} .
$$

We say that $\omega$ is Week Liouville, if $\omega \in\{\omega \mid \beta(\omega)<c\}$, where c is a positive constant depending on $\mathrm{a}(\mathrm{x})$ and $\mathrm{b}(\mathrm{x})$, and will be specified later. Then we get the following main theorem:

Main Theorem . Assume thatL $\left(E_{0}, \omega\right)>0$ and $\omega$ is Week Liouville. Then there exists $\rho>0$ such that for any $\mathrm{E}, \mathrm{E}^{\prime} \in\left(E_{0}-\rho_{0}, E_{0}+\rho_{0}\right)$ holds

$$
\left|\mathrm{L}(\mathrm{E})-\mathrm{l}\left(\mathrm{E}^{\prime}\right)\right|<\exp \left(-c|\log | E-E^{\prime}||^{\alpha}\right)
$$


where $\alpha=\alpha\left(\mathrm{a}, \mathrm{b}, \omega, E_{0}\right)>0$.

For the continuity of the Lyapunov exponent, Goldstein and Schlag developed two powerful tools, the Large Deviation Theorem and the Avalanche Principle, in [3]. Then, in [1,2,6], people were always using these two tools to prove the continuity of Lyapunov exponent in different condition. Now we all know that the Avalanche Principle is easy to be satisfied, and the Large Deviation Theorem is the key. So we can prove the Main Theorem very easily if we get the following called Large Deviation Theorem in our condition:

Theorem 1 (Large Deviation Theorem). Assume that $\mathrm{L}\left(E_{0}, \omega\right)>0$ and $\omega$ is Week Liouville. Then for any $\kappa>0$, there exists $N_{0}$, such that for any $\mathrm{N}>N_{0}$, we have

$$
\operatorname{mes}\left\{\mathrm{x} \in \mathbb{T}:\left|\frac{1}{N} \log \right|\left|M_{N}^{a}\left(x, \omega, E_{0}\right)\right|\left|-\frac{1}{N} \int_{\mathbb{T}} \log \right|\left|M_{N}^{a}\left(x, \omega, E_{0}\right)\right||d x|>\kappa\right\}<\exp \left(-c N^{\tau}\right),
$$

where c and $\tau$ are constants.

\section{Proof of the Main Theorem}

As mentioned above, we only need to prove the Large Deviation Theorem, whose proof is separated into the following several steps:

2.1. The Fourier Coefficient of the Subharmonic Function. The following theorem is Lemma 2.2 in [4], whose proof will be omitted in this paper.

Lemma 2.1. Let u $: \Omega \rightarrow \mathbb{R}$ be a subharmonic function on a domain $\Omega \subset \mathbb{C}$. Suppose that $\partial \Omega$ consists of finitely many piece-wise $C^{1}$ curves. There exists a positive measure $\mu$ on $\Omega$ such that for any $\Omega_{1} \subseteq \Omega$ (ie, $\Omega_{1}$ is a compactly contained subregion of $\Omega$ ),

$$
\mathrm{u}(\mathrm{z})=\int_{\Omega_{1}} \log |z-\xi| d \mu(\xi)+h(z),
$$

where $\mathrm{h}$ is harmonic on $\Omega_{1}$ and $\mu$ is unique with this property. Moreover, $\mu$ and h satisfy the

$$
\begin{array}{r}
\text { bounds } \mu\left(\Omega_{1}\right) \leq \mathrm{C}\left(\Omega, \Omega_{1}\right)\left({ }_{\Omega}^{\text {sup }} \mathrm{u}-{ }_{\Omega_{1}}^{\sup } \mathrm{u}\right), \\
|| h-{ }_{\Omega_{1}}^{\sup } u||_{L^{\infty}\left(\Omega_{2}\right)} \leq \mathrm{C}\left(\Omega, \Omega_{1}, \Omega_{2}\right)\left({ }_{\Omega}^{\sup } \mathrm{u}-{ }_{\Omega_{1}}^{\sup } \mathrm{u}\right)
\end{array}
$$

for any $\Omega_{2} \subseteq \Omega_{1}$.

Let

$$
\begin{gathered}
u_{N}=u_{N}(x, \omega, E)=\frac{1}{N} \log \left\|M_{N}^{a}(x, \omega, E)\right\|, \\
w_{N}=w_{N}(x, \omega, E)=\max \left(\frac{1}{N} \log \| M_{N}^{a}\left(x, \omega, E \|,-N^{r}\right),\right.
\end{gathered}
$$

where $\mathrm{r}$ is constant and to be defined later. Then $u_{N}(\omega, \mathrm{E})$ is subharmonic function on the domain $\operatorname{Imz} z_{j}<\rho$, and so is $w_{N}(\mathrm{z}, \omega, \mathrm{E})$, as the maximum of two subharmonic functions is also subharmonic. By the upper lemma, it yields

$$
\left|\widehat{u_{N}}(k)\right|,\left|\widehat{w_{N}}(k)\right|<\frac{B}{|k|},
$$

where $\hat{f}(k)$ is the k-th Fourier coefficient of $\mathrm{f}(\mathrm{x})$.

\subsection{Some Estimate with Any Frequency.}

Lemma 2.2. There exist $c_{1}>0$, s.t. for any $0<\delta<1$, there exists $C_{2}<\infty$, s.t.

$$
\operatorname{meas}\left\{\mathrm{x}:\left|w_{N}(x)-w_{N}(x+\omega)\right|>\frac{C_{2}}{N^{1-\delta}}\right\}<\exp \left(-c_{1} N^{\delta}\right),
$$


Proof of Lemma 2.2. Note that

$$
\left|w_{N}(x)-w_{N}(x+\omega)\right| \leq\left|\frac{1}{N} \log \right|\left|M_{N}^{a}(x)\right|\left|-\frac{1}{N} \log \right|\left|M_{N}^{a}(x+\omega)\right||| .
$$

There exists $C<\infty$, s.t.

$$
|| A^{a}(x+j \omega)\|<C,\| A^{a}(x+j \omega)^{-1} \| \leq \frac{1}{d(x+j \omega)} C
$$

Let $d_{j}(x)=\operatorname{det}\left(A^{a}(x+j \omega)\right)$.j $\left.\left.\omega\right)\right)$. Therefore, we have

$$
\left\|M_{N}^{a}(x+\omega)\right\|<\frac{1}{d(x)} C^{2}\left\|M_{N}^{a}(x)\right\|, \text { and } \quad\left\|M_{N}^{a}(x)\right\|<\frac{1}{\left|d_{N}(x)\right|} C^{2}\left\|M_{N}^{a}(x+\omega)\right\| .
$$

Set $0<\delta<1$. Consider the two cases: Case (a) : $\left|d_{j}\right| \geq \exp \left(-N^{\delta}\right), \mathrm{j}=0, \mathrm{~N}$ and case (b) :|d $d_{j} \mid<$ $\exp \left(-N^{\delta}\right)$ for some $\mathrm{j} \in\{0, \mathrm{~N}\}$. If we are in the case (a), then the above calculation gives:

and hence

$$
\max \left(\frac{\left\|M_{N}^{a}(x)\right\|}{\left\|M_{N}^{a}(x+\omega)\right\|}, \frac{\left\|M_{N}^{a}(x+\omega)\right\|}{\| M_{N}^{a}(x)||}\right)<\exp \left(N^{\delta}\right) C^{2},
$$

$$
\left|\frac{1}{N} \log \right|\left|M_{N}^{a}(x)\right|\left|-\frac{1}{N} \log \right|\left|M_{N}^{a}(x+\omega)\right|||<\frac{C}{N^{1-\delta}} .
$$

We now need to bound the measure for the case (b). Let $\mathbb{S}=\left\{\mathrm{x} \in \mathbb{T}:\left|d_{j}\right|<\exp \left(-N^{\delta}\right)\right.$, for some $\left.\mathrm{j} \in\{0, \mathrm{~N}\}\right\}$. By the Lojasiewicz inequality ([5]),

$$
\operatorname{meas}\{\mathrm{x} \in \mathbb{T}:|\mathrm{d}(\mathrm{x})|<\delta\}<\delta^{\alpha},
$$

for any sufficiently small $\delta$ and $\alpha$ depending only on $\mathrm{d}(\mathrm{x})$. Therefore,

$$
\operatorname{meas}(\mathbb{S})<2 \exp \left(-\alpha N^{\delta}\right)<\exp \left(-c_{1} N^{\delta}\right)
$$

Thus,

$$
\begin{aligned}
\operatorname{Meas}\left\{\mathrm{x}:\left|w_{N}(x)-w_{N}(x+j \omega)\right|>\frac{j C_{2}}{N^{1-\delta}}\right\} & <\operatorname{jexp}\left(-c_{1} N^{\delta}\right), \text { meas }\left\{\mathrm{x}:\left|w_{N}(x)-w_{R}(x)\right|>\frac{R C_{2}}{N^{1-\delta}}\right\} \\
& <2 R^{2} \exp \left(-c_{1} N^{\delta}\right) .
\end{aligned}
$$

Lemma 2.3. For $\mathrm{N}$ large enough,

$$
\left|<w_{N}>-<u_{N}>\right|<\exp \left(-c_{7} N^{r}\right) .
$$

Proof of Lemma 2.3. Set $\mathbb{X}=\left\{\mathrm{x} \in \mathbb{T}:|| M_{N}^{a}(x)||<\exp \left(-N^{1+r}\right)\right\}=\left\{x: w_{N}(x) \neq u_{N}(x)\right\}$. Then

$$
\left|<w_{N}>-<u_{N}>\right|=\frac{1}{N} \int_{\mathbb{X}}\left|\log \frac{e^{-N^{1+r}}}{\| M_{N}^{a}(x)||}\right| d x .
$$

Since $\|M\|^{2} \geq|\operatorname{det} M|$, hence if $\mathrm{x} \in \mathbb{X}$, then

$$
\prod_{j=0}^{N-1}\left|d_{j}(x)\right|<\exp \left\{-2 N^{1+r}\right\}
$$

So

$$
\mathbb{X}^{\prime}=\left\{\mathrm{x}: \prod_{j=1}^{N-1}\left|d_{j}(x)\right|<\exp \left(-2 N^{1+r}\right)\right\} \subset \mathbb{X}=\left\{\mathrm{x} \in \mathbb{T}:|| M_{N}^{a}(x)||<\exp \left(-N^{1+r}\right)\right\}
$$

As $\prod_{j=0}^{N-1}\left|d_{j}(x)\right|$ is formed by $\mathrm{N}$ part by multiplication, there will be some one such that

$\exists \mathrm{i} \in\{0,1, \cdots, \mathrm{N}-1\},\left|d_{i}(\mathrm{x})\right|<e^{-2 N^{r}}$,if $\mathrm{x} \in \mathbb{X}^{\prime}$.Set $\mathbb{S}_{i}=\left\{\mathrm{x}:\left|d_{j}(x)\right|<e^{-2 N^{r}}\right\}$,so

$$
\mathbb{X}^{\prime} \subset \bigcup_{i=0}^{N-1} \mathbb{S}_{i} \text { and meas }\left(\mathbb{X}^{\prime}\right) \leq N \mid \times \operatorname{meas}\left(\mathbb{S}_{0}\right) \text {. }
$$


By (1),

$$
\operatorname{meas}\left(\mathbb{S}_{i}\right)=\operatorname{meas}\left(\mathbb{S}_{0}\right)=\operatorname{meas}\left(\left\{\mathrm{x}:|\mathrm{d}(\mathrm{x})|<e^{-2 N^{r}}\right\}\right)<\left(e^{-2 N^{r}}\right)^{\alpha}=e^{-2 \alpha N^{r}}
$$

So

$$
\operatorname{mes} \mathbb{X}<\operatorname{meas}\left(\mathbb{X}^{\prime}\right)<N e^{-2 \alpha N^{r}}
$$

What's more, we have

\section{Lemma 2.4.}

$$
\int_{\mathbb{X} \prime^{\prime}} \log \left|\mathrm{d}_{\mathrm{j}}(\mathrm{x})\right| \mathrm{dx}<\exp \left(-\mathrm{c}_{6} \mathrm{~N}^{\mathrm{r}}\right), \forall \mathrm{j} \in\{1,2, \cdots \mathrm{N}\}
$$

Proof of Lemma 2.4. Set $\mathbb{B}_{\mathrm{k}}=\left\{\mathrm{x}: \frac{\epsilon}{2^{\mathrm{k}}} \leq\left|\mathrm{d}_{\mathrm{j}}(\mathrm{x})\right|<\frac{\epsilon}{2^{\mathrm{k}-1}}\right\}$. Then

$$
\begin{gathered}
\int_{\mathbb{X} \prime} \log \left|\mathrm{d}_{\mathrm{j}}(\mathrm{x})\right| \mathrm{dx}=\sum_{\mathrm{k}=1}^{\infty} \int_{\mathbb{B}_{\mathrm{k}} \cap \mathbb{X} \prime} \log \left|\mathrm{d}_{\mathrm{j}}(\mathrm{x})\right| \mathrm{dx}+\int_{\mathbb{X} \prime \backslash \cup_{\mathrm{k}=1}^{\infty} \mathbb{B}_{\mathrm{k}}} \log \left|\mathrm{d}_{\mathrm{j}}(\mathrm{x})\right| \mathrm{dx} \leq \\
\sum_{\mathrm{k}=1}^{\infty} \operatorname{meas}\left(\mathbb{B}_{\mathrm{k}}\right)\left|\log \frac{\epsilon}{2^{\mathrm{k}}}\right|+\operatorname{meas}\left(\mathbb{X}^{\prime}\right)|\log \epsilon| .
\end{gathered}
$$

We only consider $d_{j}(x)$ is small, as when $d_{j}(x)$ is big, we have the same result. By (1), we have

$$
\operatorname{meas}\left(\mathbb{B}_{\mathrm{k}}\right)<\left(\frac{\epsilon}{2^{\mathrm{k}-1}}\right)^{\alpha}
$$

Therefore,

$$
\begin{aligned}
\int_{\mathbb{X}^{\prime}} \log \left|\mathrm{d}_{\mathrm{j}}(\mathrm{x})\right| \mathrm{dx} & <\sum_{\mathrm{k}=1}^{\infty}\left(\frac{\epsilon}{2^{\mathrm{k}-1}}\right)^{\alpha}\left|\log \frac{\epsilon}{2^{\mathrm{k}}}\right|+\mathrm{N} \exp \left(-2 \alpha N^{r}\right)|\log \epsilon| \\
& <\left(C \epsilon^{\alpha}+\mathrm{N} \exp \left(-2 \alpha N^{r}\right)\right)|\log \epsilon|<\exp \left(-\mathrm{c}_{6} \mathrm{~N}^{\mathrm{r}}\right),
\end{aligned}
$$

by setting $\epsilon=\exp (-2 \mathrm{~N})$.

As

and by (2) and Lemma 2.4,

$$
\log \frac{e^{-N^{1+r}}}{|| M_{N}^{a}(x) \mid} \geq 0, \mathrm{X} \notin \mathbb{X}
$$

$$
\begin{aligned}
\left|<w_{N}>-<u_{N}>\right| & \leq \frac{1}{N} \int_{\mathbb{X}^{\prime}}\left|(-\mathrm{N})^{1+\mathrm{r}}-\frac{1}{2} \sum_{\mathrm{j}=0}^{\mathrm{N}-1} \log \right| d_{j}(x)|| d x \\
& \leq \frac{1}{N} \operatorname{meas}\left(\mathbb{X}^{\prime}\right) \mathrm{N}^{1+\mathrm{r}}+\left|\int_{\mathbb{X}^{\prime}} \log \right| \mathrm{d}(\mathrm{x})|\mathrm{dx}|<\exp \left(-\mathrm{c}_{7} \mathrm{~N}^{\mathrm{r}}\right) .
\end{aligned}
$$

2.3.Proof of The Large Deviation Theorem. Above all, we have

$$
\begin{gathered}
\left|u_{N}(x)-<u_{N}>\right| \leq\left|u_{N}(x)-w_{N}(x)\right|+\left|w_{N}(x)-w_{R}(x)\right|+\left|w_{R}(x)-<w_{N}>\right| \\
+\left|<w_{N}>-<u_{N}>\right|
\end{gathered}
$$

and

$$
\operatorname{mes}\left\{\mathrm{x}:\left|u_{N}(x)-w_{N}(x)\right|>\kappa\right\} \leq \operatorname{mes} \mathbb{X}<N e^{-2 \alpha N^{r}}
$$

In [5], You and Zhang proved that if $\beta(\omega)<\frac{\kappa}{\mathrm{C}}$,

Thus

$$
\operatorname{mes}\left\{\mathrm{x}:\left|w_{R}-<w_{N}>\right|>\kappa\right\}<\exp \left(-c \kappa^{3} \mathrm{R}\right) \text {. }
$$

$\operatorname{Mes}\left\{\mathrm{x}:\left|u_{N}(x)-<u_{N}>\right|>2 \kappa+\frac{R C_{2}}{N^{1-\delta}}+\exp \left(-\mathrm{c}_{2} \mathrm{~N}^{\mathrm{r}}\right)\right\}<\exp \left(-\mathrm{c \kappa}^{2} \mathrm{R}\right)+N e^{-2 \alpha N^{r}}$ 


$$
+2 \mathrm{R}^{2} \exp \left(-\mathrm{c}_{1} \mathrm{~N}^{\delta}\right)
$$

Let $\mathrm{R}=\mathrm{N}^{1-2 \delta}$ and $\kappa>\mathrm{N}^{-\delta}$, and $\delta=\mathrm{r}=0.2$, then for any $\kappa>0$, there exists $\mathrm{N}_{\mathrm{k}}>0$, such that for any $\mathrm{N}>\mathrm{N}_{\mathrm{k}}$, we have

$$
\operatorname{mes}\left\{\mathrm{x}:\left|u_{N}(x)-<u_{N}>\right|>\kappa\right\}<\exp \left(-\mathrm{cN}^{0.2}\right),
$$

So we prove the large deviation theorem and then the main theorem has been proved .

\section{Acknowledgments}

This work was financially supported by the National Nature Science Foundation of China (Grant 11401166).

\section{References}

[1] J.Bourgain and S.Jitomirskaya. Continuity of the Lyapunov exponent for quasiperiodic operators with analytic potential, J. Stat.Phys. 108(2002) 1028-1218 .

[2] J.Bourgain, M.Goldstein and W.Schlag. Anderson localization for Schrodinger operators on $\mathbb{Z}$ with potentials given by the skewshift.Commun. Math. Physics 220, (2001), 583-621 . [3] M.Goldstein and W.Schlag. Holder continuity of the integrated density of states for quasi-periodic Schrodinger equations and averages of shifts of subharmonic functions. Ann. of Math. 154, (2001), 155-203 .

[4] M.Goldstein and W.Schlag. Fine properties of the integrated density of states and a quantitative separation property of the Dirichlet eigenvalues. Geom. Funct. Analysis. 18, (2008), 755-869. [5] Lojasiewicz S.Sur le probleme de la division. Studia Math. 18, (1959), 87-136.

[6] You, J.,Zhang, S., Holder continuity of the Lyapunov exponent for analytic quasiperiodic Schr"odinger cocycles with week Liouville frequency Ergod. Th. Dynam. Sys. , 34, (2014), 1395 1408. 